\title{
26. PRELIMINARY PETROLOGY OF DIABASES FROM SITES 469 AND 471, DEEP SEA DRILLING PROJECT LEG 631
}

\author{
Tsugio Shibata, Department of Earth Sciences, Okayama University, Tsushima, Okayama 700, Japan \\ and \\ Stephen E. DeLong, Department of Geological Sciences, State University of New York at Albany, Albany, New York
}

\begin{abstract}
Diabasic rocks were recovered at Sites 469 and 471 on IPOD/DSDP Leg 63. The diabasic rocks are composed mainly of plagioclase, clinopyroxene, and low-temperature alteration products. In addition to these phases, a considerable amount of primary biotite and lesser colorless amphibole are observed in some of the Site 471 diabases. Major and trace element data suggest that these rocks are tholeiitic; however, their highly altered nature obscures their petrologic affinity with the DSDP Leg 63 tholeiitic basalts and others from the nearby Pacific ocean floor. It is likely that the Site 469 and 471 diabasic rocks represent products of off-ridge intrusive activity.
\end{abstract}

\section{INTRODUCTION}

The holes drilled at Sites 469 and 471, DSDP Leg 63, encountered highly altered, diabasic rocks at 368.5 and 742 meters, respectively, below the surface of the ocean floor. The diabase unit at Site 469 is 18.5 meters thick and separated from the underlying pillow basalt basement by approximately 5 meters of clayey nannofossil chalk. At Site 471, drilling penetrated 78 meters into this sequence of altered diabase and thin intercalated sediment layers. Both sites are coincidentally located at the base of the continental rise developed between the $\mathrm{Pa}$ cific ocean floor and the continental borderland or shelf of North America. The locations of DSDP Leg 63 drill sites are shown in Figure 1 of Shibata, DeLong, and Lyman (this volume). In this paper, we present petrographic and major and trace element data for the altered diabasic rocks recovered at Sites 469 and 471 and our preliminary interpretations of them. The methods of data acquisition employed in this study are the same as those in our petrological study of the DSDP Leg 63 basalts (Shibata, DeLong, and Lyman, this volume).

\section{PETROGRAPHY AND MINERAL CHEMISTRY}

\section{Site 469}

The lithologic summary of the diabasic unit at Site 469 is shown in Figure 1. This unit is composed mainly of fine- to medium-grained, aphyric diabase with microcrystalline basalt at the base and, although not recovered, probably at the top of this unit. The chilled zone of basalt at the base is gradational upward into diabase. The gradual increase of grain size takes place within an interval of 1.5 meters from the base. The rest of the recovered portion of this unit is macroscopically quite similar in appearance, and no significant variation is detected with unaided eyes except for ill-defined, thin

\footnotetext{
${ }^{1}$ Initial Reports of the Deep Sea Drilling Project, Volume 63.
}

patches or layers that are slightly more enriched in irregular-shaped, dark green spots 1 to $3 \mathrm{~mm}$ in diameter. Thin-section analysis indicates that these dark green spots are aggregates of alteration products composed mainly of brownish green or green clays (smectite) that fill the interstices between plagioclase laths and equant pyroxenes.

The chilled margin basalt is characterized by a subvariolitic texture with skeletal plagioclase microlites, opaque minerals, and a trace amount of clinopyroxene. The basalt specimen is extremely altered with abundant greenish brown clay occurring at the interstices between plagioclase microlites. Neither fresh olivines nor olivine pseudomorphs are found. If olivine was originally present, it must have occurred as microlites in the altered mesostasis.

The diabase unit of Site 469 is composed (in approximately decreasing order of abundance) of plagioclase, clinopyroxene, brownish green clay, opaque minerals, calcite, zeolites, talc, quartz, and amphibole. The overall texture, except for that of the chilled margin basalt described above, is intersertal to intergranular. Euhedral to subhedral plagioclase and subhedral to anhedral, equant clinopyroxene ranging in grain size from 0.1 to $2 \mathrm{~mm}$ and 0.05 to $1 \mathrm{~mm}$, respectively, occur throughout and are the main primary constituents forming more than $70 \%$ of this unit. Representative microprobe analyses of clinopyroxenes from the Site 469 diabases are listed in Table 1, and all analyses are plotted in the pyroxene quadrilateral (Fig. 2). The clinopyroxenes from the Site 469 diabases show a rather restricted range of composition and are $\mathrm{Mg}$-rich augites. Some of these clinopyroxenes show faint purplish tints, generally thought to be a characteristic of titaniferous clinopyroxenes. These pyroxenes, however, are not enriched in titanium (Fig. 3). Plagioclases are strongly zoned from approximately $\mathrm{An}_{65-77}$ at the core to $\mathrm{An}_{55-60}$ at the rim (Table 2). Systematic, vertical variations of plagioclase and clinopyroxene compositions throughout the diabase unit were not observed. Although there is no 


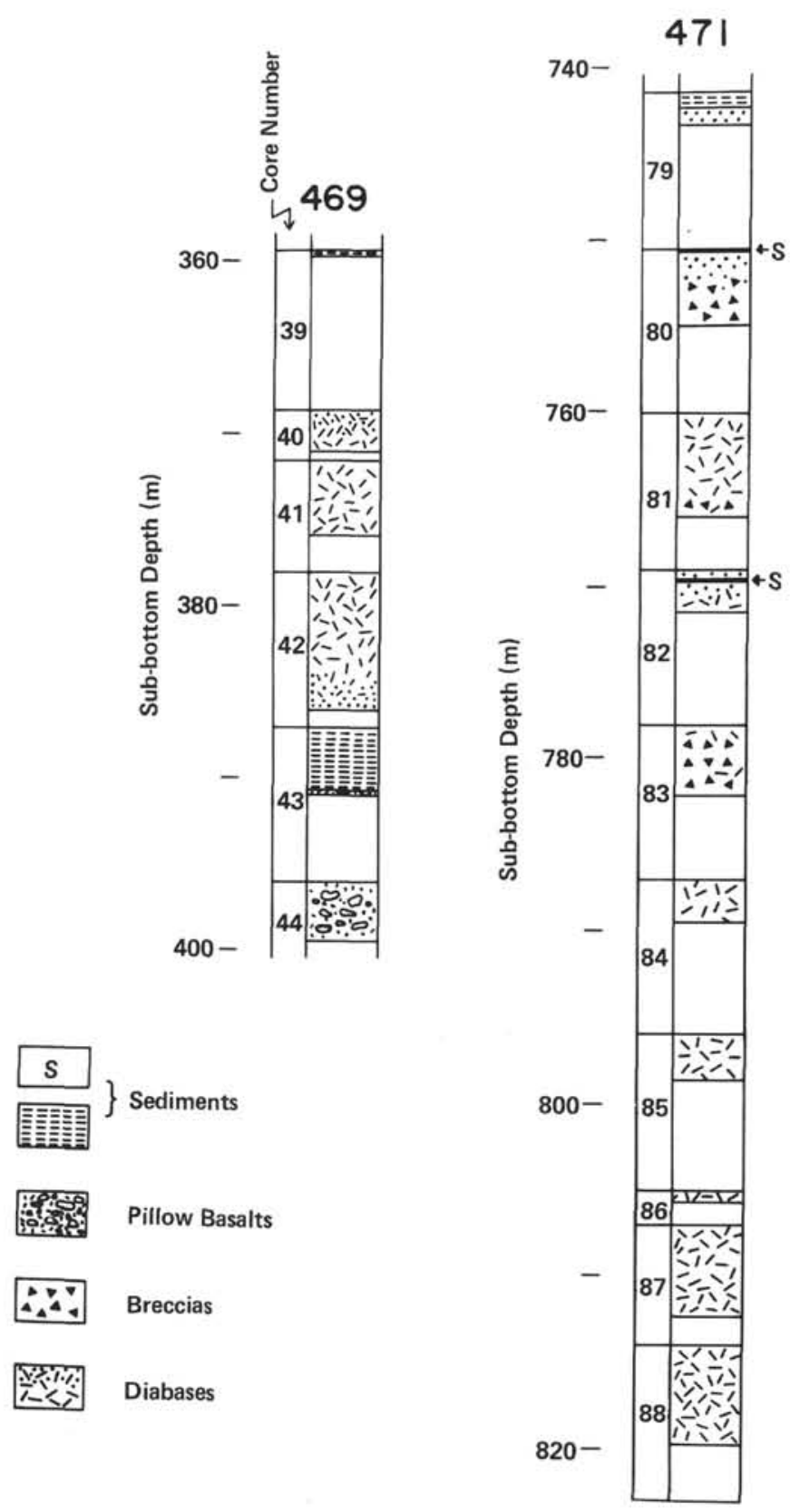

Figure 1. Lithologic summary of the diabase units of Sites 469 and 471 , DSDP Leg 63 . (The depths are in meters from the sediment/ seawater interface.)

significant change in the modal proportions of the main primary constituents throughout, quartz does occur rarely in the interstices at the top portion of this unit.

Brownish green clay minerals are another of the most abundant phases found throughout the diabase unit. These minerals occur in close association with necessary calcite and occasionally zeolites, talc, mica, and amphibole. These minerals probably formed through lowtemperature seawater alteration or late-stage deuteric processes.

\section{Site 471}

The lithologic summary of the diabase sequence at Site 471 is shown in Figure 1. Two main rock types were recovered: (1) diabase and its fine-grained equivalent, and (2) brecciated diabase, which is mainly composed of diabasic fragments (Plate 1, Figs. 1 and 2). The brecciated diabases were recovered at intervals in Cores 80 , 81 , and 83 (Fig. 1). Aphyric, fine-grained basalt occurs at the top contact (Core 79) with the overlying sediments; from this contact, grain size gradually increases downward. Two sediment layers were found at the top portions of Cores 80 and 82 . Although the immediate contact zones with the intercalated thin sediments were not recovered, the diabases are finer at the contact zones with these sediment layers, becoming slightly coarser away from them. This gradual increase of grain size takes place within an interval of 1 meter.

Thin-section analyses indicate that the specimens from Core 79 are intensely altered and have abundant calcite, clay minerals, and zeolite $(>50 \%)$ replacing or altering the original constituents. Alteration is so intense that mafic primary minerals are not found, nor any pseudomorph after them, and plagioclase is the only primary constituent remaining. The highly altered, medium-grained diabases that constitute the main parts of the Hole 471 diabase sequence are characterized by an intersertal to subophitic texture with subhedral plagioclase and clinopyroxene up to $4 \mathrm{~mm}$ in diameter (Plate 2, Fig. 1). The interstices between these minerals are commonly filled with clay, zeolite, and/or calcite.

The pyroxene and plagioclase analyses from Site 471 are listed in Tables 1 and 2 , and are compared with those of Site 469 diabases and Leg 63 tholeiitic basalts in Figures 2, 3, and 4 . In addition to these minerals, a considerable amount of biotite $(<0.5 \mathrm{~mm})$ and lesser amphibole and quartz occur in some samples from the lower portions of this hole (Cores $84,85,86,87$, and 88 ) (Plate 2, Figs. 2 and 3). The amphiboles are acicular and nearly colorless with faint greenish tint, and the biotites are commonly zoned from the brownish core to the greenish rim. The microprobe analyses of these minerals are listed in Table 3.

The brecciated diabases are made up of angular to subangular rock fragments set in a fine-grained matrix. The macroscopic texture of these rocks is shown in Plate 1, Figures 1 and 2, and a photomicrograph of cryptocrystalline fragments is shown in Plate 2, Figure 4. The rock fragments are generally less than $2 \mathrm{~cm}$ across and include altered diabase, its fine-grained equivalent, and sediments (clayey chert). Also, some brecciated diabases, particularly those from Core 83 , show a laminated fabric that resembles closely that of cataclastic rocks. Their constituent minerals do not show, however, microscopic strain effects such as marginal granulation, undulatory extinction, and bending of cleavage cracks or twin lamellae that are commonly observed in cataclastic rocks. Thus it is difficult to explain this 
Table 1. Representative microprobe analyses of clinopyroxene.

\begin{tabular}{|c|c|c|c|c|c|c|c|c|c|c|c|c|}
\hline Analysis No. ${ }^{a}$ & 1 & 2 & 3 & 4 & 5 & 6 & 7 & 8 & 9 & 10 & 11 & 12 \\
\hline $\mathrm{SiO}_{2}$ & 50.01 & 52.73 & 52.73 & 51.26 & 52.63 & 50.17 & 50.13 & 50.50 & 50.05 & 50.18 & 50.31 & 49.92 \\
\hline $\mathrm{TiO}_{2}$ & 1.52 & 0.45 & 0.46 & 0.80 & 0.48 & 1.65 & 1.53 & 1.11 & 1.27 & 1.01 & 0.72 & 0.81 \\
\hline $\mathrm{Al}_{2} \mathrm{O}_{3}$ & 3.54 & 1.75 & 2.13 & 3.25 & 1.58 & 4.00 & 4.28 & 2.08 & 2.51 & 3.89 & 2.89 & 3.02 \\
\hline $\mathrm{FeO}^{*}$ & 7.89 & 5.22 & 5.93 & 6.44 & 5.22 & 7.56 & 6.44 & 13.20 & 11.70 & 7.64 & 6.75 & 6.91 \\
\hline $\mathrm{MnO}$ & 0.29 & 0.23 & 0.21 & 0.26 & 0.21 & 0.24 & 0.22 & 0.48 & 0.39 & 0.22 & 0.22 & 0.22 \\
\hline $\mathrm{MgO}$ & 15.41 & 17.80 & 17.55 & 17.12 & 17.75 & 14.71 & 15.53 & 13.84 & 14.33 & 14.71 & 16.78 & 16.34 \\
\hline $\mathrm{CaO}$ & 20.22 & 21.32 & 21.26 & 20.36 & 21.27 & 21.81 & 22.32 & 19.29 & 19.74 & 21.26 & 20.65 & 21.24 \\
\hline $\mathrm{Na}_{2} \mathrm{O}$ & 0.29 & 0.18 & 0.21 & 0.25 & 0.17 & 0.37 & 0.32 & 0.35 & 0.39 & 0.29 & 0.23 & 0.21 \\
\hline Total & 99.17 & 99.68 & 100.48 & 99.74 & 99.31 & 100.51 & 100.77 & 100.85 & 100.38 & 99.20 & 98.55 & 98.67 \\
\hline \multicolumn{13}{|c|}{ Formula basis $=6$ oxygens } \\
\hline $\mathrm{Si}$ & 1.872 & 1.937 & 1.927 & 1.891 & 1.941 & 1.857 & 1.844 & 1.901 & 1.883 & 1.878 & 1.887 & 1.876 \\
\hline $\mathrm{Al}$ & 0.156 & 0.076 & 0.092 & 0.141 & 0.069 & 0.175 & 0.186 & 0.092 & 0.111 & 0.172 & 0.128 & 0.134 \\
\hline $\mathrm{Ti}$ & 0.043 & 0.012 & 0.013 & 0.022 & 0.013 & 0.046 & 0.042 & 0.031 & 0.036 & 0.028 & 0.020 & 0.023 \\
\hline $\mathrm{Mg}$ & 0.860 & 0.975 & 0.956 & 0.941 & 0.975 & 0.812 & 0.852 & 0.776 & 0.804 & 0.821 & 0.938 & 0.915 \\
\hline $\mathrm{Fe}^{2+}$ & 0.247 & 0.160 & 0.181 & 0.199 & 0.161 & 0.234 & 0.198 & 0.415 & 0.368 & 0.239 & 0.212 & 0.217 \\
\hline $\mathrm{Mn}$ & 0.009 & 0.007 & 0.006 & 0.008 & 0.007 & 0.008 & 0.007 & 0.015 & 0.012 & 0.007 & 0.007 & 0.007 \\
\hline $\mathrm{Ca}$ & 0.811 & 0.839 & 0.832 & 0.805 & 0.840 & 0.865 & 0.880 & 0.778 & 0.796 & 0.853 & 0.830 & 0.855 \\
\hline $\mathrm{Na}$ & 0.021 & 0.013 & 0.015 & 0.018 & 0.012 & 0.027 & 0.023 & 0.026 & 0.028 & 0.021 & 0.017 & 0.015 \\
\hline Total & 4.018 & 4.019 & 4.002 & 4.025 & 4.018 & 4.023 & 4.032 & 4.035 & 4.039 & 4.018 & 4.038 & 4.042 \\
\hline $\mathrm{Ca}$ & 42.3 & 42.5 & 42.3 & 41.4 & 42.5 & 45.3 & 45.6 & 39.5 & 40.4 & 44.6 & 41.9 & 43.0 \\
\hline $\mathrm{Mg}$ & 44.8 & 49.4 & 48.6 & 48.4 & 49.3 & 42.5 & 44.1 & 39.4 & 40.9 & 42.9 & 47.4 & 46.0 \\
\hline $\mathrm{Fe}$ & 12.9 & 8.1 & 9.2 & 10.2 & 8.1 & 12.2 & 10.3 & 21.1 & 18.7 & 12.5 & 10.7 & 10.9 \\
\hline
\end{tabular}

Note: $\mathrm{FeO}^{*}=$ total iron as $\mathrm{FeO}$.

a Analysis numbers designate the following samples: $1: 469-40-2,12-18 \mathrm{~cm}$ (core). $2: 469-40-2,12-18 \mathrm{~cm} .3: 469-42-1,65-70 \mathrm{~cm}$ (core). 4: $469-42-5,70-75 \mathrm{~cm} .5: 469-42-5,70-75 \mathrm{~cm} .6: 471-81-1,15-21 \mathrm{~cm}$ (rim). 7: 471-81-1, 15-21 cm (core). 8: 471-85-1, 72-80 cm (rim). 9: $471-85-1,72-80 \mathrm{~cm}$ (core), the same grain as analysis No. 8. 10: 471-87-2, 78-87 cm (core). 11: 471-88-3, 69-78 cm (core). 12: 471-88-3, 69-78 cm (core).

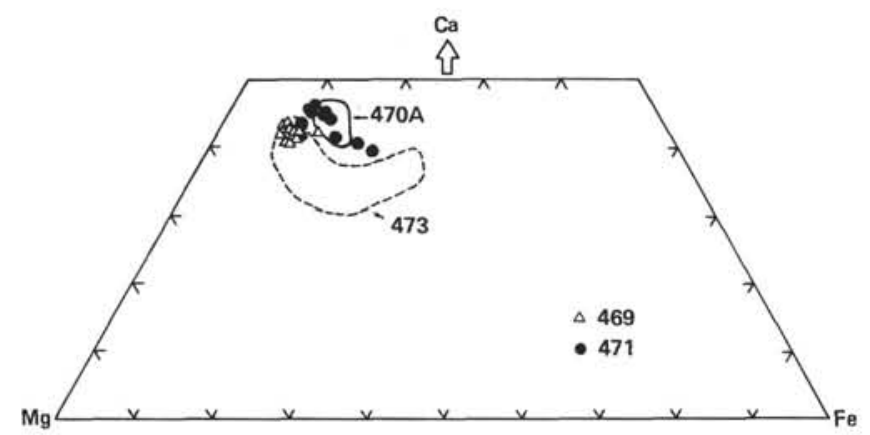

Figure 2. Pyroxene compositions in atomic Ca-Mg-Fe proportions. (The fields for the clinopyroxenes of the DSDP Leg 63 tholeiitic basalts [Shibata, DeLong, and Lyman, this volume] are enclosed by a solid line [Site 470A] and a broken line [Site 473], respectively.)

laminated fabric in terms of cataclasis in which solid diabase has been ground up and milled along a shear zone between moving rock masses. The fact that crystal fragments of plagioclase are rarely found in some of the laminated breccias, however, does imply some clastic process of formation. Although the ultimate origin of brecciation is uncertain, it is possible that these brecciated diabases could have formed in more than one way. For example, the brecciated diabase shown in Plate 1, Figure 1 could have formed by intense weathering along incipient fractures; others could have formed because of autobrecciation, i.e., a fragmentation process whereby portions of the first-consolidated crusts of

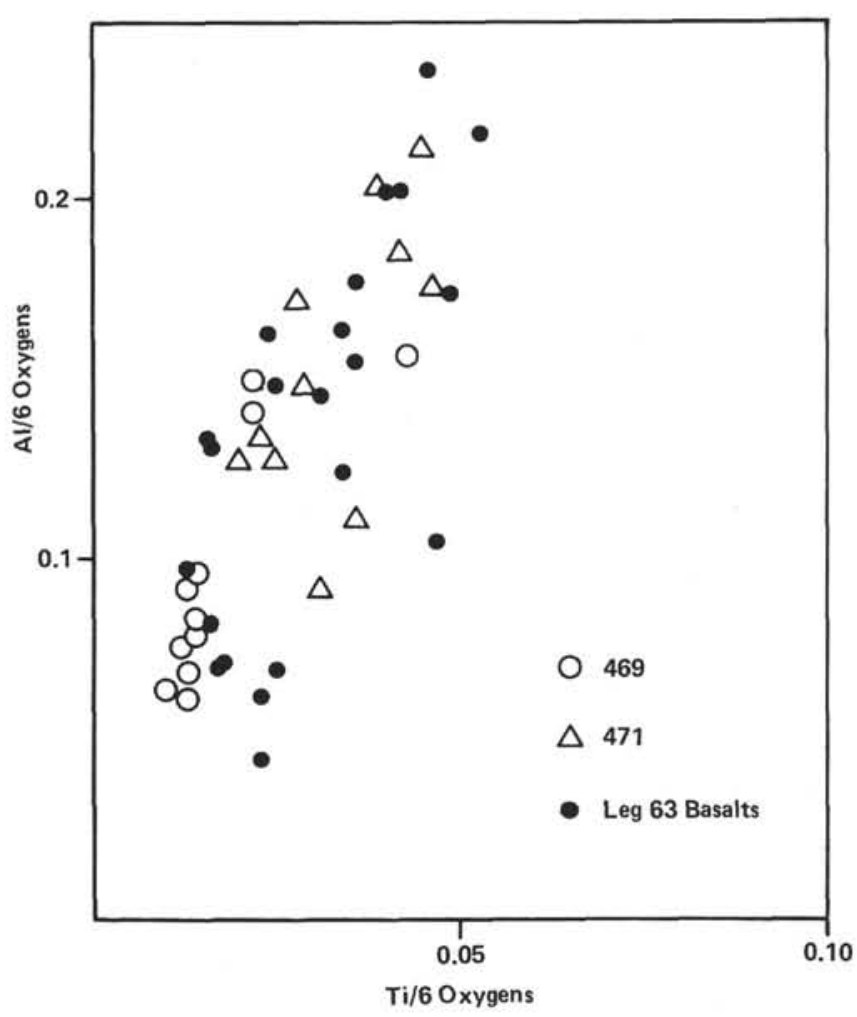

Figure 3. $\mathrm{Al}$ versus $\mathrm{Ti}$ in clinopyroxenes (atomic abundances on formula basis of 6 oxygens). (Open circles indicate Site 469 , triangles, Site 471; filled circles, pyroxenes from the DSDP Leg 63 basalts [Shibata, DeLong, and Lyman, this volume].) 
Table 2. Microprobe analyses of plagioclase.

\begin{tabular}{|c|c|c|c|c|c|c|c|c|c|c|c|c|c|c|c|}
\hline Analysis No. ${ }^{\mathrm{a}}$ & 1 & 2 & 3 & 4 & 5 & 6 & 7 & 8 & 9 & 10 & 11 & 12 & 13 & 14 & 15 \\
\hline $\mathrm{SiO}_{2}$ & 49.80 & 52.72 & 54.33 & 48.22 & 51.19 & 51.52 & 52.92 & 56.45 & 56.98 & 52.79 & 49.18 & 49.76 & 50.63 & 48.64 & 48.10 \\
\hline $\mathrm{TiO}_{2}$ & 0.07 & 0.06 & 0.12 & 0.06 & 0.08 & 0.07 & 0.08 & 0.11 & 0.12 & 0.13 & 0.06 & 0.09 & 0.03 & 0.07 & 0.06 \\
\hline $\mathrm{Al}_{2} \mathrm{O}_{3}$ & 31.30 & 29.88 & 28.76 & 33.23 & 30.50 & 29.46 & 28.27 & 27.18 & 26.61 & 29.13 & 31.39 & 30.55 & 30.68 & 31.80 & 31.57 \\
\hline $\mathrm{FeO}^{*}$ & 0.30 & 0.37 & 0.47 & 0.32 & 0.44 & 0.35 & 0.37 & 0.51 & 0.49 & 0.65 & 0.44 & 0.46 & 0.55 & 0.45 & 0.41 \\
\hline $\mathrm{MnO}$ & 0.01 & 0.04 & 0.01 & 0.03 & 0.03 & 0.04 & 0.03 & 0.02 & 0.00 & 0.03 & 0.04 & 0.02 & 0.02 & 0.02 & 0.02 \\
\hline $\mathrm{MgO}$ & 0.18 & 0.19 & 0.08 & 0.19 & 0.13 & 0.19 & 0.24 & 0.08 & 0.06 & 0.14 & 0.33 & 0.29 & 0.26 & 0.25 & 0.25 \\
\hline $\mathrm{CaO}$ & 14.34 & 13.16 & 11.04 & 16.28 & 13.29 & 14.03 & 12.85 & 9.51 & 8.91 & 12.04 & 15.10 & 15.03 & 14.76 & 15.72 & 15.16 \\
\hline $\mathrm{Na}_{2} \mathrm{O}$ & 3.43 & 3.65 & 5.03 & 2.57 & 4.10 & 3.81 & 4.42 & 6.16 & 6.41 & 4.72 & 2.93 & 2.93 & 3.29 & 2.72 & 3.00 \\
\hline $\mathrm{K}_{2} \mathrm{O}$ & 0.09 & 0.10 & 0.17 & 0.09 & 0.12 & 0.11 & 0.11 & 0.30 & 0.33 & 0.16 & 0.10 & 0.09 & 0.12 & 0.09 & 0.10 \\
\hline Total & 99.52 & 100.17 & 100.01 & 100.99 & 99.88 & 99.59 & 99.29 & 100.32 & 99.91 & 99.78 & 99.58 & 99.22 & 100.34 & 99.76 & 98.67 \\
\hline \multicolumn{16}{|c|}{ Formula basis $=8$ oxygens } \\
\hline $\mathrm{Si}$ & 2.286 & 2.387 & 2.455 & 2.194 & 2.336 & 2.360 & 2.423 & 2.537 & 2.566 & 2.405 & 2.262 & 2.294 & 2.308 & 2.237 & 2.237 \\
\hline $\mathrm{Al}$ & 1.693 & 1.595 & 1.532 & 1.782 & 1.640 & 1.591 & 1.526 & 1.439 & 1.412 & 1.565 & 1.702 & 1.660 & 1.648 & 1.725 & 1.730 \\
\hline $\mathrm{Ti}$ & 0.002 & 0.002 & 0.004 & 0.002 & 0.003 & 0.002 & 0.003 & 0.004 & 0.004 & 0.005 & 0.002 & 0.003 & 0.001 & 0.002 & 0.002 \\
\hline $\mathrm{Mg}$ & 0.012 & 0.013 & 0.005 & 0.013 & 0.009 & 0.013 & 0.016 & 0.005 & 0.004 & 0.009 & 0.023 & 0.020 & 0.018 & 0.017 & 0.017 \\
\hline $\mathrm{Fe}^{2+}$ & 0.012 & 0.014 & 0.018 & 0.012 & 0.017 & 0.013 & 0.014 & 0.019 & 0.018 & 0.025 & 0.017 & 0.018 & 0.021 & 0.017 & 0.016 \\
\hline $\mathrm{Mn}$ & 0.000 & 0.002 & 0.000 & 0.001 & 0.001 & 0.001 & 0.001 & 0.001 & 0.000 & 0.001 & 0.002 & 0.001 & 0.001 & 0.001 & 0.001 \\
\hline $\mathrm{Ca}$ & 0.705 & 0.638 & 0.535 & 0.794 & 0.650 & 0.689 & 0.630 & 0.458 & 0.430 & 0.588 & 0.744 & 0.743 & 0.721 & 0.775 & 0.755 \\
\hline $\mathrm{Na}$ & 0.305 & 0.320 & 0.441 & 0.227 & 0.363 & 0.339 & 0.392 & 0.537 & 0.560 & 0.417 & 0.262 & 0.262 & 0.291 & 0.243 & 0.271 \\
\hline K & 0.005 & 0.006 & 0.010 & 0.005 & 0.007 & 0.007 & 0.007 & 0.017 & 0.019 & 0.009 & 0.006 & 0.005 & 0.007 & 0.005 & 0.006 \\
\hline Total & 5.021 & 4.977 & 5.000 & 5.029 & 5.026 & 5.015 & 5.012 & 5.017 & 5.013 & 5.024 & 5.020 & 5.006 & 5.016 & 5.022 & 5.035 \\
\hline An mole $\%$ & 69.5 & 66.2 & 54.3 & 77.4 & 63.7 & 66.6 & 61.2 & 45.3 & 42.6 & 58.0 & 73.5 & 73.6 & 70.8 & 75.8 & 73.2 \\
\hline
\end{tabular}

Note: $\mathrm{FeO}^{*}=$ total iron as $\mathrm{FeO}$.

a Analysis numbers designate the following samples: 1: 469-40-2, 12-18 cm (core). 2: 469-40-2, 12-18 cm (core). 3: 469-40-2, 12-18 cm (rim). 4: $469-42-1,65-70 \mathrm{~cm}$ (core). 5: 469-42-1, 65-70 cm (rim), the same grain as Analysis No. 4. 6: 469-42-5, 70-75 cm (core). 7: 469-42-5, 70-75 cm (rim). $8: 471-81-1,15-21 \mathrm{~cm}$ (core). 9: 471-81-1, 15-21 cm (rim), the same grain as Analysis No. 8. 10: 471-85-1, 72-80 cm (core). 11: 471-85-1, 72-80 cm (core). 12: 471-87-2, 78-87 cm (core). 13: 471-87-2, 78-87 cm (rim), the same grain as Analysis No. 12. 14: 471-88-3, 69-78 cm (core). 15: 471-88-3, 69-78 cm (core).

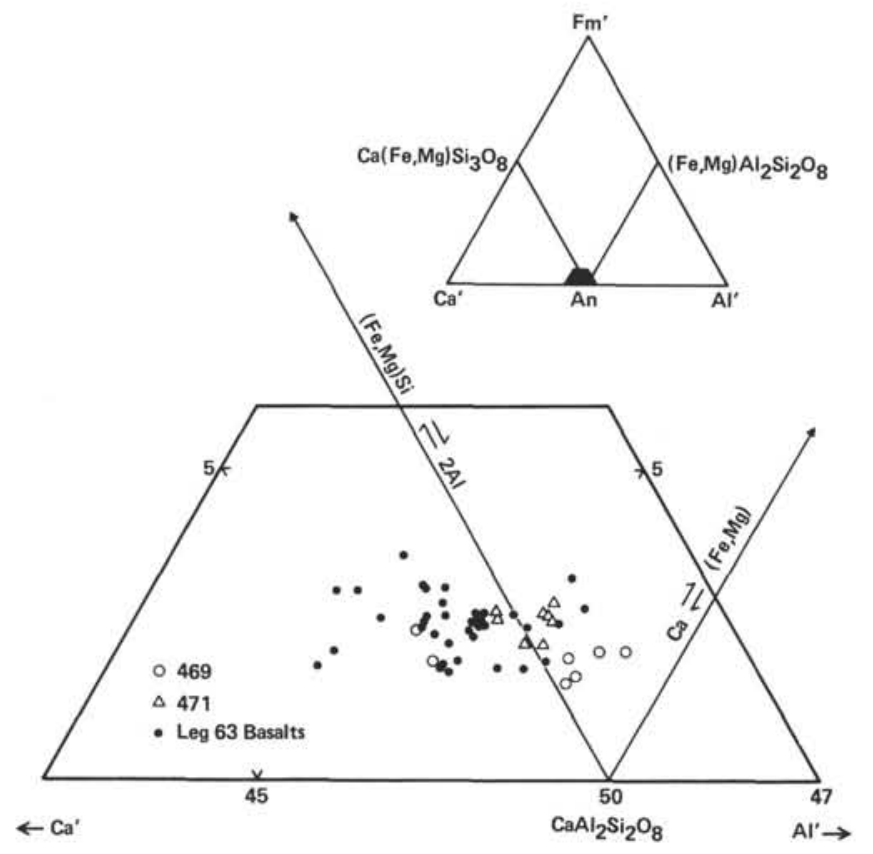

Figure 4. Plagioclase compositions in the plane $\mathrm{Fm}^{\prime}\left[(\mathrm{Fe}, \mathrm{Mg})_{2} \mathrm{Si}_{3} \mathrm{O}_{8}\right]-$ $\mathrm{Ca}^{\prime}\left(\mathrm{Ca}_{2} \mathrm{Si}_{3} \mathrm{O}_{8}\right)-\mathrm{Al}^{\prime}\left(\mathrm{Al}_{4} \mathrm{SiO}_{8}\right)$ (Longhi, 1976; Longhi et al., 1976). (Open circles indicate Site 469, triangles, Site 471, and filled circles, plagioclases from the DSDP Leg 63 basalts [Shibata, DeLong, and Lyman, this volume].)
Table 3. Microprobe analyses of amphibole and biotites.

\begin{tabular}{lrrrrr}
\hline Analysis No. $^{\mathrm{a}}$ & \multicolumn{1}{c}{1} & \multicolumn{1}{c}{2} & \multicolumn{1}{c}{3} & \multicolumn{1}{c}{4} & \multicolumn{1}{c}{5} \\
\hline $\mathrm{SiO}_{2}$ & 46.54 & 38.85 & 38.40 & 36.18 & 38.56 \\
$\mathrm{TiO}_{2}$ & 1.20 & 4.91 & 3.98 & 2.38 & 2.05 \\
$\mathrm{Al}_{2} \mathrm{O}_{3}$ & 6.31 & 12.49 & 11.62 & 12.21 & 11.68 \\
$\mathrm{FeO}^{*}$ & 16.18 & 14.84 & 14.94 & 15.32 & 14.33 \\
$\mathrm{MnO}$ & 0.36 & 0.12 & 0.09 & 0.19 & 0.13 \\
$\mathrm{MgO}$ & 13.11 & 15.38 & 15.54 & 18.53 & 17.89 \\
$\mathrm{CaO}$ & 10.20 & 0.11 & 0.10 & 0.12 & 0.09 \\
$\mathrm{Na} 2 \mathrm{O}$ & 2.19 & 0.99 & 0.76 & 0.25 & 0.47 \\
$\mathrm{~K} 2 \mathrm{O}$ & 0.52 & 8.08 & 8.14 & 4.27 & 7.04 \\
$\mathrm{Total}$ & 96.61 & 95.77 & 93.57 & 89.44 & 92.23 \\
Formula basis $=$ & 23 & 22 & 22 & 22 & 22 \\
oxygens & & & & & \\
$\mathrm{Si}$ & 7.005 & 5.742 & 5.822 & 5.636 & 5.865 \\
$\mathrm{Al}$ & 1.120 & 2.176 & 2.078 & 2.243 & 2.094 \\
$\mathrm{Ti}$ & 0.136 & 0.546 & 0.453 & 0.279 & 0.234 \\
$\mathrm{Mg}$ & 2.940 & 3.389 & 3.511 & 4.302 & 4.055 \\
$\mathrm{Fe} 2+$ & 2.037 & 1.834 & 1.894 & 1.996 & 1.823 \\
$\mathrm{Mn}$ & 0.046 & 0.016 & 0.012 & 0.025 & 0.016 \\
$\mathrm{Ca}$ & 1.646 & 0.018 & 0.016 & 0.020 & 0.015 \\
$\mathrm{Na}$ & 0.640 & 0.283 & 0.225 & 0.076 & 0.139 \\
$\mathrm{~K}$ & 0.101 & 1.524 & 1.575 & 0.849 & 1.366 \\
$\mathrm{Total}$ & 15.671 & 15.527 & 15.585 & 15.426 & 15.606 \\
& & & & &
\end{tabular}

Note: $\mathrm{FeO}^{*}=$ total iron as $\mathrm{FeO}$.

a Analysis numbers designate the following samples: 1: Hornblende, 471-87-2, 78-87 cm. 2: Biotite, 471-87-2, 78-87 cm. 3: Biotite, 471-87-2, 78-87 cm. 4: Biotite (greenish rim), 471$88-3,69-78 \mathrm{~cm}$. 5: Biotite, 471-88-3, 69-78 cm. 
intrusions or flows are incorporated into the still-molten interior.

\section{WHOLE-ROCK CHEMISTRY}

Major and trace element data of the diabasic rocks (Tables 4 and 5) are apparently modified through intense low-temperature alteration processes, as indicated by their high $\mathrm{K}_{2} \mathrm{O}$ and $\mathrm{Rb}$ contents and ignition loss. This makes it difficult to specify the original whole-rock chemistry and to define the magmatic affinity of the diabases. All of the analyzed rocks except one (Sample 471-81-2, 43-51 cm) show olivine + hypersthene or hypersthene + quartz in their norms (Table 4). Although the number of analyses is small, these rocks do not show clearly defined tholeiitic trends in the AFM diagram (Fig. 5). In the alkali-silica diagram (not shown), two samples (469-42-6, 27-32 cm and 471-81-2,
43-51 cm) plot in the Hawaiian alkaline field, but the rest of the diabasic rocks plot in the tholeiitic field (Macdonald and Katsura, 1964). Al and Ti concentrations in clinopyroxenes are known to be sensitive to the degree of silica saturation of their host basalt magmas (Kushiro, 1960; LeBas, 1962), among other factors. Figure 3 indicates that the $\mathrm{Al}$ and $\mathrm{Ti}$ concentrations in the clinopyroxenes of the Site 469 and 471 diabasic rocks are quite similar to those of the DSDP Leg 63 tholeiitic basalts (Shibata, DeLong, and Lyman, this volume), suggesting a tholeiitic character for the host diabasic rocks. (The Leg 63 diabasic clinopyroxenes are essentially indistinguishable on this plot, however, from those in ocean-floor alkaline rocks, e.g., Site 169 of DSDP Leg 17, Myers et al., 1975). None of the above results unambiguously define the magma type(s) of the Site 469 and 471 diabasic rocks; but considering the

Table 4. Major element compositions and norms of DSDP Leg 63 diabases.

\begin{tabular}{|c|c|c|c|c|c|c|c|c|c|}
\hline $\begin{array}{c}\text { Sample } \\
\text { (interval in } \mathrm{cm} \text { ) }\end{array}$ & $\begin{array}{c}469-40-2 \\
12-18\end{array}$ & $\begin{array}{c}469-41-3 \\
98-103\end{array}$ & $\begin{array}{c}469-42-5 \\
70-75\end{array}$ & $\begin{array}{c}469-42-6 \\
27-32\end{array}$ & $\begin{array}{l}\begin{array}{l}471-79-1, \\
125-130\end{array}\end{array}$ & $\begin{array}{c}471-81-2, \\
43-51\end{array}$ & $\begin{array}{c}471-85-1 \\
72-80\end{array}$ & $\begin{array}{c}471-87-2, \\
78-87\end{array}$ & $\begin{array}{c}471-88-3, \\
69-78\end{array}$ \\
\hline $\mathrm{SiO}_{2}$ & 51.27 & 52.44 & 51.54 & 49.35 & 28.99 & 49.63 & 50.67 & 47.88 & 43.29 \\
\hline $\mathrm{TiO}_{2}^{2}$ & 1.31 & 1.41 & 1.30 & 1.38 & 1.45 & 2.00 & 1.46 & 1.24 & 1.02 \\
\hline $\mathrm{Al}_{2} \mathrm{O}_{3}$ & 15.68 & 15.06 & 15.15 & 16.51 & 12.17 & 14.73 & 14.77 & 16.06 & 14.26 \\
\hline $\mathrm{Fe}_{2} \mathrm{O}_{3}$ & 3.54 & 3.29 & 3.74 & 4.97 & 1.46 & 3.09 & 2.86 & 2.40 & 3.09 \\
\hline $\mathrm{FeO}$ & 4.19 & 4.75 & 4.36 & 5.90 & 5.64 & 5.42 & 5.85 & 6.83 & 5.74 \\
\hline $\mathrm{FeO}^{*}$ & 7.38 & 7.71 & 7.73 & 10.37 & 6.95 & 8.20 & 8.42 & 8.99 & 8.52 \\
\hline $\mathrm{MnO}$ & 0.11 & 0.11 & 0.10 & 0.16 & 1.14 & 0.50 & 0.22 & 0.20 & 0.26 \\
\hline $\mathrm{MgO}$ & 6.87 & 6.95 & 6.90 & 5.83 & 5.72 & 6.81 & 7.12 & 8.63 & 13.30 \\
\hline $\mathrm{CaO}$ & 8.18 & 8.04 & 7.11 & 4.68 & 19.08 & 5.26 & 8.45 & 10.25 & 7.59 \\
\hline $\mathrm{Na}_{2} \mathrm{O}$ & 3.73 & 3.68 & 3.43 & 3.02 & 2.44 & 4.72 & 2.49 & 2.19 & 1.59 \\
\hline $\mathrm{K}_{2} \mathrm{O}$ & 0.64 & 0.35 & 0.67 & 1.68 & 0.42 & 1.90 & 0.75 & 0.35 & 0.18 \\
\hline $\mathrm{H}_{2} \mathrm{O}^{-}$ & 2.26 & 2.15 & 2.84 & 2.59 & 1.10 & 0.77 & 2.39 & 1.31 & 4.26 \\
\hline Ig. loss & 1.97 & 1.74 & 2.12 & 3.47 & 19.60 & 4.88 & 2.87 & 2.59 & 5.06 \\
\hline Total & 99.75 & 99.97 & 99.26 & 99.54 & 99.21 & 99.71 & 99.90 & 99.93 & 99.64 \\
\hline $\mathrm{FeO} * / \mathrm{MgO}$ & 1.07 & 1.11 & 1.12 & 1.78 & 1.22 & 1.20 & 1.18 & 1.04 & 0.64 \\
\hline \multicolumn{10}{|l|}{ CIPW Norms $^{\mathrm{a}}$} \\
\hline $\mathrm{C}$ & & & & 1.21 & & & & & \\
\hline Q & & 1.93 & 2.16 & 1.25 & & & 3.65 & & \\
\hline ne & & & & & & 2.38 & & & \\
\hline or & 3.78 & 2.07 & 3.96 & 9.93 & & 11.23 & 4.43 & 2.07 & 1.06 \\
\hline ab & 31.56 & 31.14 & 29.02 & 25.55 & & 35.54 & 21.07 & 18.53 & 13.45 \\
\hline an & 24.15 & 23.54 & 23.96 & 23.22 & & 13.39 & 26.91 & 32.96 & 31.24 \\
\hline ol & 1.76 & & & & & 14.87 & & 4.84 & 15.16 \\
\hline hy & 16.12 & 19.06 & 21.08 & 26.34 & & & 21.04 & 18.10 & 19.69 \\
\hline $\mathrm{di}$ & 13.27 & 13.21 & 9.15 & & & 10.29 & 12.17 & 14.47 & 5.13 \\
\hline $\mathrm{mt}$ & 2.18 & 2.28 & 2.29 & 3.07 & & 2.43 & 2.49 & 2.66 & 2.52 \\
\hline il & 2.49 & 2.68 & 2.47 & 2.62 & & 3.80 & 2.77 & 2.36 & 1.94 \\
\hline
\end{tabular}

Note: $\mathrm{FeO}^{*}=$ total iron as $\mathrm{FeO}$.

a The norms were calculated after $\mathrm{Fe}_{2} \mathrm{O}_{3}$ was reduced to $\mathrm{FeO}$ so as to have $\mathrm{Fe}_{2} \mathrm{O}_{3} / \mathrm{FeO}=0.25$.

Table 5. Trace-element abundances of DSDP Leg 63 diabases.

\begin{tabular}{cccccccccc}
\hline $\begin{array}{c}\text { Sample } \\
\text { (interval in cm) }\end{array}$ & $\begin{array}{c}469-40-2, \\
12-18\end{array}$ & $\begin{array}{c}469-41-3, \\
98-103\end{array}$ & $\begin{array}{c}469-42-5, \\
70-75\end{array}$ & $\begin{array}{c}469-42-6, \\
27-32\end{array}$ & $\begin{array}{c}471-79-1, \\
125-130\end{array}$ & $\begin{array}{c}471-81-2, \\
43-51\end{array}$ & $\begin{array}{c}471-85-1, \\
72-80\end{array}$ & $\begin{array}{c}471-87-2, \\
78-87\end{array}$ & $\begin{array}{c}471-88-3, \\
69-78\end{array}$ \\
\hline $\mathrm{Rb}$ & 15.3 & 5.2 & 12.6 & 16.9 & 13.3 & 28.6 & 9.1 & 6.3 & 3.5 \\
$\mathrm{Sr}$ & 193 & 199 & 189 & 166 & 115 & 110 & 176 & 165 & 124 \\
$\mathrm{Y}$ & 25.0 & 23.4 & 29.5 & 15.1 & 28.0 & 29.4 & 25.0 & 21.3 & 15.1 \\
$\mathrm{Zr}$ & 112 & 116 & 113 & 114 & 110 & 138 & 121 & 76.1 & 59.0 \\
$\mathrm{Nb}$ & $<5$ & $<5$ & $<5$ & $<5$ & 10.8 & 16.0 & 12.8 & 8.7 & 6.2 \\
$\mathrm{~V}$ & 178 & 175 & 181 & 192 & 205 & 237 & 248 & 216 & 199 \\
$\mathrm{Cr}$ & 42.8 & 33.7 & 39.5 & 47.3 & 185 & 175 & 110 & 224 & 214 \\
$\mathrm{Cr}$ & 48.3 & 44.3 & 45.4 & 34.3 & 30.7 & 37.1 & 53.8 & 57.7 & 55.3 \\
$\mathrm{Ni}$ & 14.8 & 13.7 & 15.7 & 28.9 & 85.7 & 59.6 & 70.5 & 152 & 205 \\
$\mathrm{Cu}$ & 11.4 & 4.2 & 8.9 & 13.2 & 27.9 & 26.5 & 47.9 & 31.9 & 29.7 \\
$\mathrm{Zn}$ & 57.2 & 59.2 & 59.8 & 62.2 & 42.7 & 52.1 & 53.6 & 44.4 & 45.7 \\
\hline
\end{tabular}




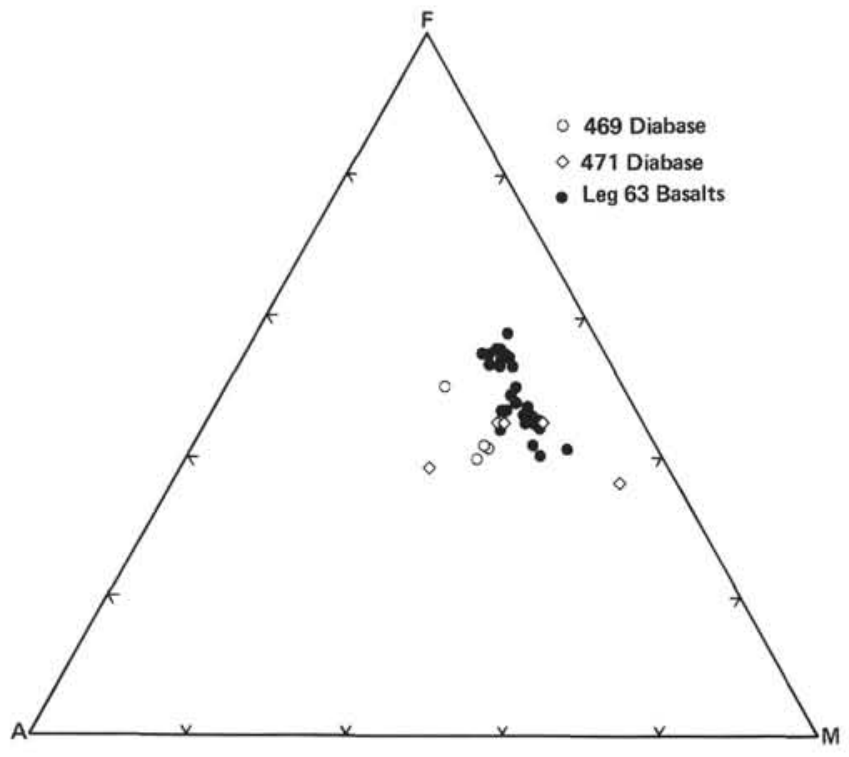

Figure 5. AFM diagram, $\left(\mathrm{Na}_{2} \mathrm{O}+\mathrm{K}_{2} \mathrm{O}\right)$ - $\mathrm{FeO}$ - $\mathrm{MgO}$, showing the distribution of DSDP Leg 63 diabasic rocks. (Open circles, Site 469; open squares, Site 471; filled circles, DSDP Leg 63 tholeiites and basalt glasses [Shibata, DeLong, and Lyman, this volume].)

highly altered nature of these rocks, we suspect that the Site 469 and 471 diabasic rocks were most likely derived from tholeiitic magma(s).

Variations with depth of oxide concentration and $\mathrm{FeO}^{*} / \mathrm{MgO}$ are shown in Figures 6 and 7, respectively, for Sites 469 and 471 . One remarkable feature in these diagrams is that the samples from the contact zones with overlying or underlying sediments differ significantly in composition from other samples. Sample 469-42-6, $27-32 \mathrm{~cm}$ is more enriched in $\mathrm{FeO}^{*}$ and $\mathrm{Al}_{2} \mathrm{O}_{3}$ relative to other Site 469 samples, and Sample 471-79-1, 125-130 $\mathrm{cm}$, showing substantial ignition loss, is more enriched in $\mathrm{CaO}$. Although we do not have sediment analyses from the immediate contact zone overlying or underlying the diabasic rocks, one plausible interpretation for the unusual rock compositions would be contamination with the sediments. For example, the rock composition of Sample 471-79-1, 125-130 cm could be generated by the contamination of other Site 471 rocks with calcareous ooze or limestone.

\section{DISCUSSION}

Investigators who have studied samples from DSDP drill holes have been faced with difficulties in determining the mode of occurrence of massive, coarse-grained basaltic rocks that lack pillow structure. In most cases, the contact relations of these massive basaltic rocks with the surrounding lithologic units and the three-dimensional configuration of the igneous bodies are unknown (Hall and Robinson, 1979). As at earlier sites, the recovery of the uppermost portions of the diabase units at Sites 469 and 471 is very poor. We interpret the Site 469 and 471 diabasic rocks, however, to be intrusive. The evidence supporting this view is: (1) the absence of pillow structure and microscopic quench texture that would indicate rapid chilling of magma against cold seawater; (2) the relatively coarse grain size of the diabasic rocks compared to basaltic pillows and lava flows; (3) the significant changes of oxide abundances in the samples from the contact zones relative to other samples in the same cores; and (4) the presence of sedimentary rock fragments in the Site 471 brecciated diabases.

We infer that the diabasic rocks are probably tholeiitic, although their highly altered nature obscures their petrologic affinity with abyssal tholeiites. Apparently, these diabasic rocks were intruded into thick sediments. Because there is generally only thin or no sediment cover on the basaltic basement at oceanic ridge crests, this observation and the apparent intrusive origin of the diabasic rocks may indicate that they are products of off-ridge igneous activity.

\section{ACKNOWLEDGMENTS}

We thank A. Minami of Hiroshima University for the microprobe analyses presented in this report; T. Saito (Okayama) and P. Lyman (Albany) for technical assistance; and J. F. Bender and D. J. Fornari for critical comments. This work was supported by the Japan Society for the Promotion of Science and the U.S. National Science Foundation (Grants OCE76-21882 and INT78-22980).
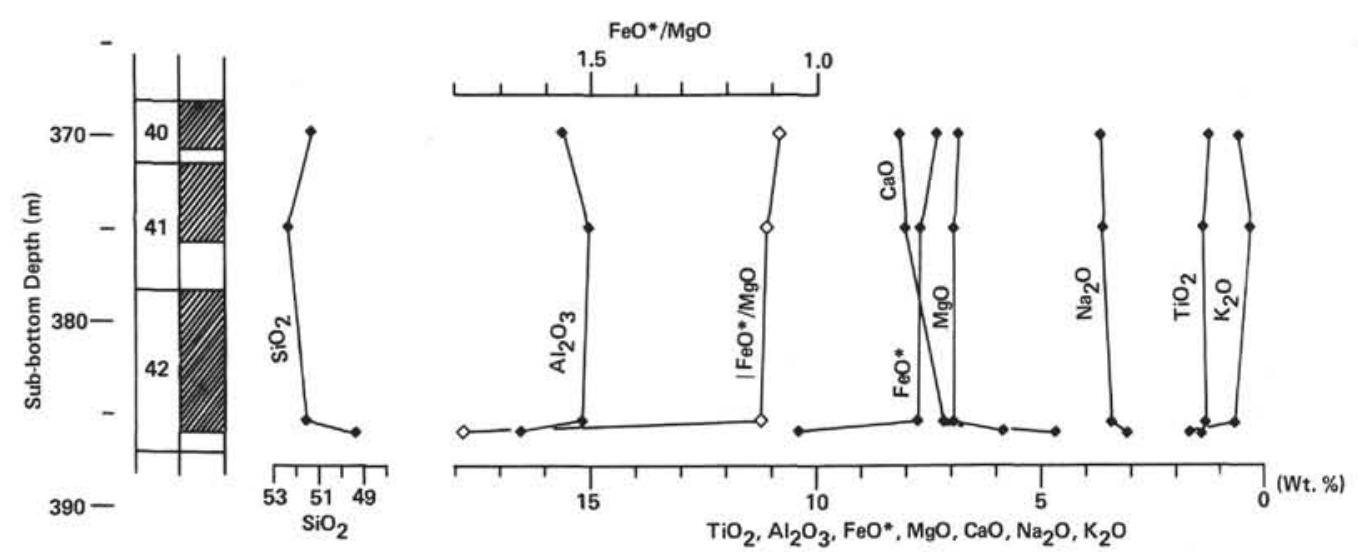

Figure 6. Oxide abundances and $\mathrm{FeO}^{*} / \mathrm{MgO}$ variation versus depth at Site 469, DSDP Leg 63. (The depth is in meters from the sediment/seawater interface.) 


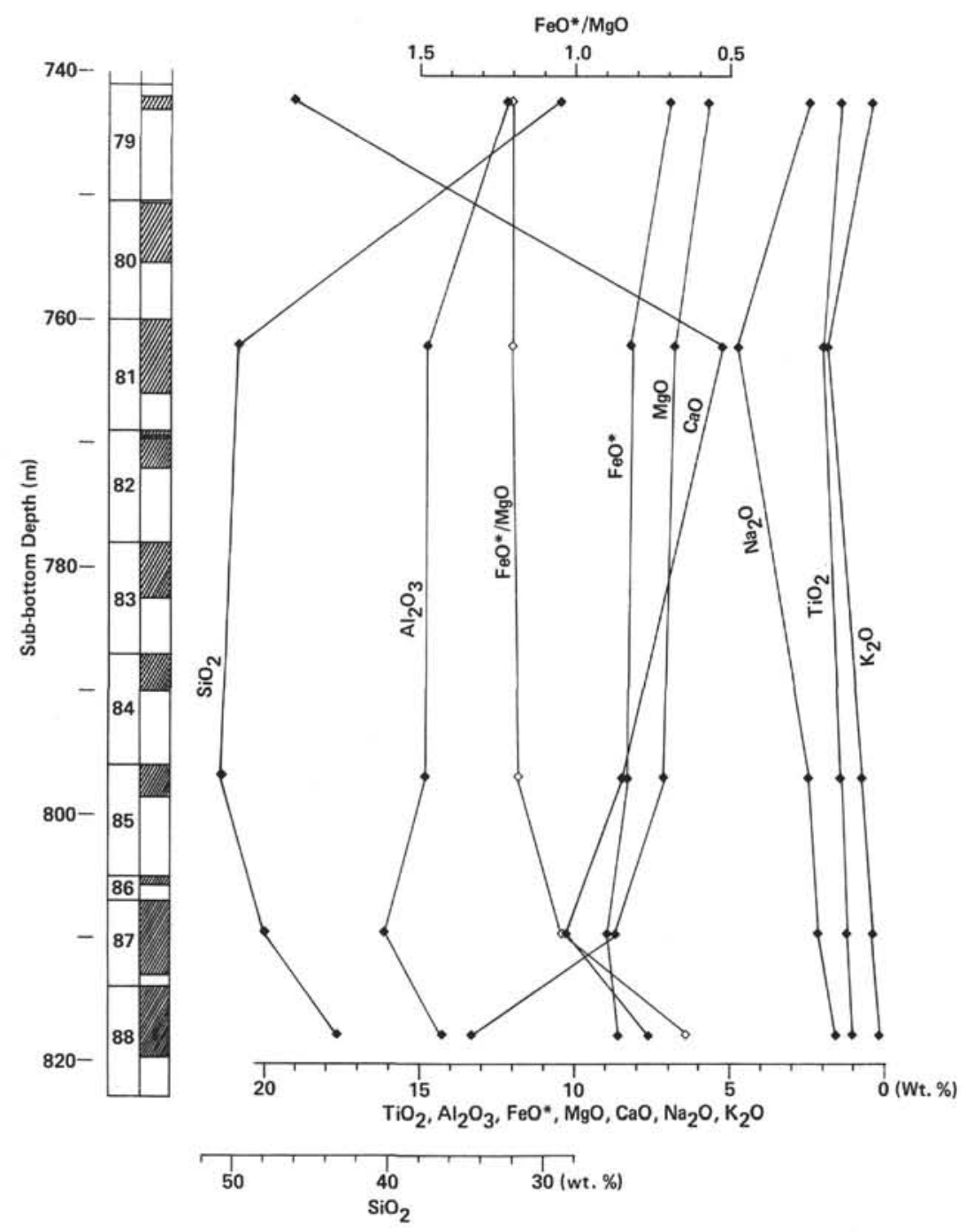

Figure 7. Oxide abundance and $\mathrm{FeO} * / \mathrm{MgO}$ variation versus depth at Site 471, DSDP Leg 63. (The depth is in meters from the sediment/seawater interface.)

\section{REFERENCES}

Hall, J. M., and Robinson, P. T., 1979. Deep crustal drilling in the north Atlantic ocean. Science, 204:573-586.

Kushiro, I., 1960. Si-Al relations in clinopyroxenes from igneous rocks. Am. J. Sci., 258:548-554.

LeBas, M. J., 1962. The role of aluminum in igneous clinopyroxenes with relation to their parentage. Am. J. Sci., 260:267-288.
Longhi, J., 1976. Iron, magnesium and silica in plagioclase [Ph.D. thesis]. Harvard University.

Longhi, J., Walker, D., and Hays, J. F., 1976. Fe and Mg in plagioclase. Proc. Seventh Lunar Sci. Conf., pp. 1281-1300.

Macdonald, G. A., and Katsura, T., 1964. Chemical composition of Hawaiian lavas. J. Petrol., 5:82-133.

Myers, C. W., Bence, A. E., Papike, J. J., et al., 1975. Petrology of an alkali-olivine basalt sill from Site 169 of DSDP Leg 17: the Central Pacific basin. J. Geophys. Res., 80:807-822. 

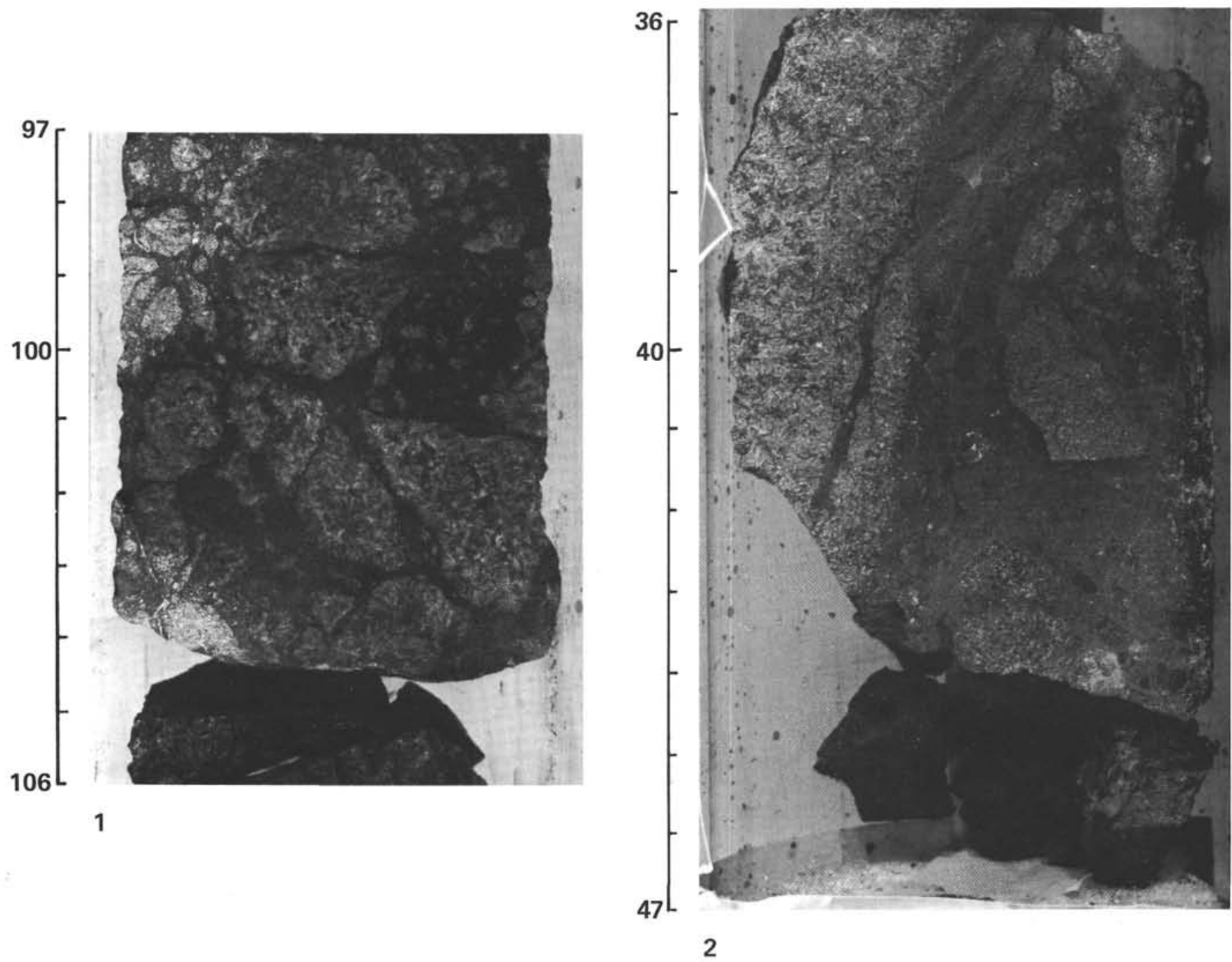

Plate 1. 1. Brecciated diabase. Sample 471-80-2, 97-106 cm. 2. Brecciated diabase. Sample 471-80-3, 35-47 cm. (Note dark-colored, angular chert fragments $[<5 \mathrm{~mm}]$.) 

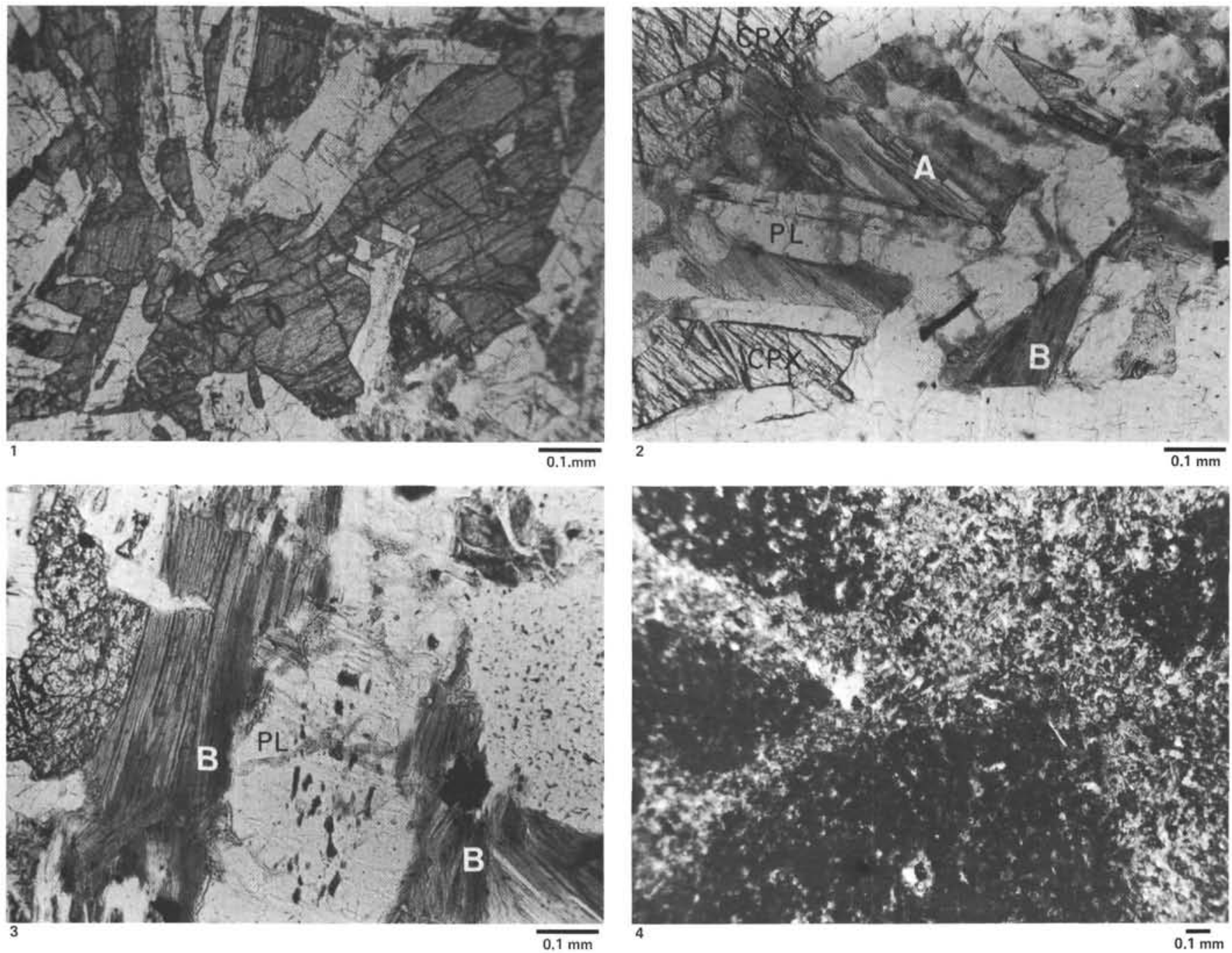

Plate 2. 1. Subophitic texture in Sample 471-87-1, 113-117 cm (plane light). 2, 3. Photomicrograph (in plane light) of diabase containing biotite and amphibole. Sample 471-88-2, 89-92 cm. (B: biotite; A: amphibole; CPX: clinopyroxene; PL: plagioclase.) 4. Photomicrograph (in plane light) of brecciated diabase containing cryptocrystalline rock fragments. Sample 471-82-1, 165-168 cm. 